Journal of Computational and Applied Mechanics, Vol. 12., No. 2., (2017), pp. 99-107 DOI: $10.32973 /$ jcam.2017.007

\title{
UNSTEADY MHD FREE CONVECTIVE HEAT AND MASS TRANSFER FLOW WITH VARIABLE HEAT AND MASS FLUXES
}

\author{
Chavi Maheshwari, Govind Pathak \\ Department of Mathematics, Radha Govind Engineering College \\ Garh Road, Meerut (U.P), 250004, India \\ chhavi.rathi@gmail.com, pathakgovind@Rediffmail.com
}

S. P. GUPTA

B.S.A. College, Mathura (U.P), India

[Received: March 15, 2017; Accepted: July 12, 2017]

\begin{abstract}
The mass, momentum and heat transfer in unsteady free convention flow along an infinite porous vertical plate, suddenly set in uniform motion with variable heat and mass fluxes at the plate, in the presence of uniform transverse magnetic field fixed to the plate, has been investigated. Similar solutions of energy and concentration boundary layer equations, for a particular form of suction/injection velocity $v_{w}(t)$, is obtained. For solution of the momentum boundary layer equation, a series expansion of velocity function in powers of product of magnetic field parameter and time is assumed. The analytical solutions of resulting ordinary differential equations have been obtained in terms of repeated integrals of complementary error functions. Also, the velocity, temperature and concentration profiles have been drawn and discussed.
\end{abstract}

Keywords: boundary layer flow, free convection, heat and mass flux, mass transfer, skin friction coefficient

\section{INTRODUCTION}

Elbashbeshy [1] and Pohlhausen 2] have free convection flow of fluids past a semiinfinite isothermal vertical plate in presence of magnetic field. Mohsin and Mandal 3], Dave et al. [4 and Pathak and Tak [5] have studied the unsteady free convection flow along an accelerated infinite porous plate, in presence of a transverse magnetic field, fixed relative to fluid. Soundalgekar [6], Tak and Pathak [7] and Tak and Sisodia [8] studied the same problem for the magnetic field fixed relative to the plate. In the present problem unsteady mass, momentum and heat transfer are investigated in free convection flow of binary fluids along a vertical porous plate suddenly set in uniform motion in its own plane, with variable heat and mass fluxes in presence of transverse magnetic field fixed to the plate. 


\section{MATHEMATICAL FORMULATION AND ANALYSIS}

Consider an unsteady flow of an incompressible electrically conducting fluid in the presence of foreign species along an infinite vertical porous non-conducting plate in presence of uniform transverse magnetic field. At time $t^{\prime} \leq 0$ the temperature and foreign species concentration at the plate and the fluid are assumed to be $T_{\infty}^{\prime}$ and $c_{\infty}^{\prime}$, respectively. For $t^{\prime} 0$, the plate temperature and species concentration at the plate are instantaneously raised such that there is a variable heat flux $q_{\infty}^{\prime}$ per unit area and variable mass flux $m_{w}$ per unit area from the plate moves upwards in its own plane and is maintained at uniform velocity $u_{0}^{\prime}$. The transverse magnetic field is assumed to be fixed relative to the plate. The axis $x^{\prime}$ is taken along the plate in upward direction upward direction and the axis $y^{\prime}$ is normal to it. For a small concentration level, the Soret-Dufour effects can be neglected in the energy equation. The electrical conductivity of the fluid is assumed to be small so that the induced magnetic field can be neglected in comparison of applied magnetic field. Then, under the usual Boussinesq's approximations, equations governing the flow in absence of frictional heating are (Gebhart [9]):

$$
\begin{gathered}
\frac{\partial v^{\prime}}{\partial y^{\prime}}=0 \Rightarrow v^{\prime}=v_{\omega}^{\prime}\left(t^{\prime}\right) \\
\frac{\partial u^{\prime}}{\partial t^{\prime}}+v^{\prime} \frac{\partial u^{\prime}}{\partial y^{\prime}}=v \frac{\partial^{2} u^{\prime}}{\partial y^{\prime 2}}+g \beta\left(T^{\prime}-T_{\infty}^{\prime}\right)+g \beta^{*}\left(c^{\prime}-c_{\infty}^{\prime}\right)-\frac{\sigma B_{0}^{2}\left(u^{\prime}-u_{0}^{\prime}\right)}{\rho} \\
\frac{\partial T^{\prime}}{\partial t^{\prime}}+v^{\prime} \frac{\partial T^{\prime}}{\partial y^{\prime}}=\frac{k}{\rho c_{p}} \frac{\partial^{2} T^{\prime}}{\partial y^{\prime 2}} \\
\frac{\partial c^{\prime}}{\partial t^{\prime}}+v^{\prime} \frac{\partial c^{\prime}}{\partial y^{\prime}}=D \frac{\partial^{2} c^{\prime}}{\partial y^{\prime 2}}
\end{gathered}
$$

where $u^{\prime}$ and $v^{\prime}$ are longitudinal and normal components of velocity respectively, $\nu$ is the kinematic viscosity, $T^{\prime}$ is the temperature, $c^{\prime}$ is the concentration, $D$ is the coefficient of mass diffusion, $g$ is the gravitational acceleration, $\beta$ is the coefficient of thermal expansion, $\beta^{*}$ is the coefficient of species concentration expansion, $k$ the thermal conductivity, $c_{p}$ is the specific heat at constant pressure, $\sigma$ is the electrical conductivity, $\rho$ is the density and $B_{0}$ is the uniformly applied magnetic field strength.

The initial and boundary conditions are:

$$
\left.\begin{array}{l}
t^{\prime} \leq 0: u^{\prime}=0, T^{\prime}=T_{\infty}^{\prime}, \quad c^{\prime}=c_{\infty}^{\prime} \forall y^{\prime} \\
t^{\prime} \leq 0: u^{\prime}=0, T^{\prime}=T_{\infty}^{\prime}, \quad c^{\prime}=c_{\infty}^{\prime} \forall y^{\prime} \\
\frac{\partial c^{\prime}}{\partial y^{\prime}}=-\frac{m_{w}^{\prime}(t)}{D}=-\frac{c_{\infty}^{\prime}}{2 \sqrt{\nu t^{\prime}}} \text { at } y^{\prime}=0 \\
u^{\prime}=u_{0}^{\prime}\left(1-e^{-m_{1} t^{\prime}}\right), T^{\prime}=T_{\infty}^{\prime}, \quad c^{\prime}=c_{\infty}^{\prime} \text { as } y^{\prime} \rightarrow \infty
\end{array}\right\}
$$

where $m_{1}=\sigma B_{0}^{2} / \rho$. The last boundary condition on velocity is obtained by integrating equation (2) at the boundary layer edge, where

$$
\frac{\partial u^{\prime}}{\partial y^{\prime}}=\frac{\partial^{2} u^{\prime}}{\partial y^{\prime 2}}=0, \quad T^{\prime}=T_{\infty}^{\prime}, \quad c^{\prime}=c_{\infty}^{\prime} .
$$


Introducing the following non-dimensional quantities:

$$
\begin{gathered}
y=\frac{y^{\prime} u_{0}^{\prime}}{v}, \quad t=\frac{t^{\prime} u_{0}^{\prime 2}}{v}, \quad u=\frac{u^{\prime}}{u_{0}^{\prime}}, \quad v=\frac{v^{\prime}}{u_{0}^{\prime}}, \quad v_{w}=\frac{v_{w}^{\prime}}{u_{0}^{\prime}}, \quad \theta=\frac{T^{\prime}-T_{\infty}^{\prime}}{T_{\infty}^{\prime}}, \quad c=\frac{c^{\prime}-c_{\infty}^{\prime}}{c_{\infty}^{\prime}} \\
G r=\frac{v g \beta T_{\infty}^{\prime}}{u_{0}^{\prime 3}} \text { (Grashof numbers) }, \quad m=\frac{\sigma B_{0}^{2} v}{\rho u_{0}^{\prime 2}} \text { (Magnetic parameter), } \\
G c=\frac{v g \beta^{*} c_{\infty}^{\prime}}{u_{0}^{\prime 3}} \text { (Modified Grashof number), } \operatorname{Pr}=\frac{\mu c_{p}}{\kappa} \text { (Prandtl number) }, \\
S c=\frac{v}{D} \text { (Schmidt number) }
\end{gathered}
$$

and then applying the following transformations:

$$
\left.\begin{array}{l}
u=\sum_{i=0}^{\infty}(m t)^{i} f_{i}(\eta), \theta=\theta(\eta), c=c(\eta), \\
\eta=\frac{y}{2 \sqrt{t}}, \quad v_{w}(t)=-\frac{a}{\sqrt{t}}
\end{array}\right\}
$$

equations (2) and (4) are reduced to the following set of ordinary linear differential equations:

$$
\begin{gathered}
\theta^{\prime \prime}+2 \operatorname{Pr}(a+\eta) \theta^{\prime}=0, \\
c^{\prime \prime}+2 S c(a+\eta) c^{\prime}=0, \\
f_{0}^{\prime \prime}+2(a+\eta) f_{0}^{\prime}=0, \\
f_{1}^{\prime \prime}+2(a+\eta) f_{1}^{\prime}-4 f_{1}-4 f_{0}+\frac{4 G r \theta}{m}+\frac{4 G c c}{m}+4=0, \\
f_{i}^{\prime \prime}+2(a+\eta) f_{i}^{\prime}-4 . i . f_{i}-4 f_{i-1}=0, \quad i \geq 2,
\end{gathered}
$$

which are associated with the following initial and boundary conditions:

$$
\left.\begin{array}{l}
\eta=0: \theta^{\prime}=-1, c^{\prime}=-1, f_{0}=1, f_{i}=0 \quad \forall i \geq 1 \\
\eta \rightarrow \infty: \theta=0, c=0, f_{0}=0, f_{1}=1, f_{2}=\frac{-1}{2 !}, f_{i}=(-1)^{i-1} \frac{1}{i !}, i \geq 2
\end{array}\right\}
$$

where $a$ is the suction/injection parameter. It may be noted that for suction $a>0$, for injection $a<0$ and for impermeable plate $a=0$.

The homogeneous parts of the above system of differential equation admit solutions in terms of repeated integrals of the complementary error functions (See Abramowitz and Stegun [10]). For the non-homogeneous part of equation 10 the particular integrals have been obtained by the method of undetermined coefficients. The complete solutions of equation $(7)$ to $(10$, , satisfying boundary conditions $\sqrt{12}$ are as follows:

$$
\begin{gathered}
\theta(\sqrt{\operatorname{Pr}} \xi)=\frac{1}{\sqrt{\operatorname{Pr}}} \frac{i^{0} \operatorname{erf} f_{c}(\sqrt{\operatorname{Pr}} \xi)}{i^{-1} \operatorname{erf} f_{c}(\sqrt{\operatorname{Pr}} a)}, \quad \xi=a+\eta, \\
C=\frac{1}{\sqrt{S c}} \frac{i^{0} \operatorname{erf} f_{c}(\sqrt{S c} \xi)}{i^{-1} \operatorname{erf} f_{c}(\sqrt{S c} a)}, \\
f_{0}(\xi)=\frac{i^{0} \operatorname{erf} f_{c}(\xi)}{i^{0} \operatorname{erf}(a)},
\end{gathered}
$$




$$
\begin{aligned}
f_{1}(\xi)=A i^{2} \operatorname{erf} f_{c}(\xi)-\frac{i^{0} e r f_{c}(\xi)}{i^{0} e r f_{c}(a)}-\frac{4 G r i^{2} e r f_{c}(\sqrt{\operatorname{Pr}} \xi)}{\sqrt{\operatorname{Pr}} m(\operatorname{Pr}-1) i^{-1} \operatorname{erf} f_{c}(\sqrt{\operatorname{Pr}} a)}- \\
-\frac{4 G c \cdot i^{2} \operatorname{erf} f_{c}(\sqrt{S c} \xi)}{\sqrt{S c} m(S c-1) i^{-1} \operatorname{erf} f_{c}(\sqrt{S c} a)}+1,
\end{aligned}
$$

where

$$
\begin{aligned}
& A=\frac{4 G r i^{2} \operatorname{erf} f_{c}(\sqrt{\operatorname{Pr}} a)}{m \sqrt{\operatorname{Pr}}(\operatorname{Pr}-1) i^{2} \operatorname{erf} f_{c}(a) i^{-1} \operatorname{erf}(\sqrt{\operatorname{Pr}} a)}+ \\
& \quad+\frac{4 G c i^{2} \operatorname{erf} f_{c}(\sqrt{S c} a)}{m \sqrt{S c}(S c-1) i^{2} \operatorname{erf} f_{c}(a) i^{-1} \operatorname{erf} f_{c}(\sqrt{S c} a)}
\end{aligned}
$$

It may be noted that solution of equation 16 is valid when $\operatorname{Pr} \neq 1$ and $S c \neq 1$. In case of $\operatorname{Pr}=1$ and $S c=1$ by taking the limiting values we get

$$
f_{1}=C_{1} i^{2} \operatorname{erf} f_{c}(\xi)+D_{1} \operatorname{erf} f_{c}(\xi)+1
$$

where

$$
\left.\begin{array}{rl}
C_{1} & =-\frac{G r}{m} \frac{i^{0} e r f_{c}(a)}{i^{-1} \operatorname{erf} f_{c}(a) i^{2} e r f_{c}(a)}-\frac{G c}{m} \frac{i^{0} \operatorname{erf} f_{c}(a)}{i^{-1} \operatorname{erf} f_{c}(a) i^{2} e r f_{c}(a)}, \\
D_{1} & =\frac{1}{i^{-1} \operatorname{erf}(a)}\left[\frac{G r+G c}{m}\right]-\frac{1}{i^{0} \operatorname{erf}(a)} .
\end{array}\right\}
$$

and the function $i^{n} \operatorname{er} f_{c}(\xi)$ is the repeated integral of complementary error function defined as:

$$
\left.\begin{array}{c}
i^{n} \operatorname{erf}_{c}(\xi)=\frac{2}{\sqrt{\pi}} \int_{\xi}^{\infty} \frac{(t-\xi)^{n}}{n !} e^{-t^{2}} d t, \quad n=0,1,2 \ldots \ldots \\
=\sum_{K=0}^{\infty} \frac{(-1)^{K} \xi^{K}}{2^{n-K} K ! \Gamma\left(1+\frac{n-K}{2}\right)} \\
i^{-1} \operatorname{erf}_{c}(\xi)=\frac{2}{\sqrt{\pi}} e^{-\xi^{2}}, i^{n} \operatorname{erf}(\xi)=e r f_{c}(\xi), \\
\frac{\partial}{\partial \xi} i^{n} \operatorname{erf} f_{c}(\xi)=-i^{n-1} \operatorname{erf} f_{c}(\xi)
\end{array}\right\}
$$

and the recurrence relation is

$$
i^{n-2} \operatorname{erf} f_{c}(\xi)-2 \xi i^{n-1} \operatorname{erf} f_{c}(\xi)-2 n i^{n} \operatorname{erf} f_{c}(\xi)=0 .
$$

It is remarked here that the analytical solutions up to first order approximation have been obtained and higher order terms may be obtained in a similar manner. As the numerical values of functions like $\operatorname{erf}(\sqrt{P r} a)$ for arbitrary values of $P r, S c$ and parameter $a$ are not readily available, the differential equations (7) to (11), up to second order with boundary conditions 12 have also been solved numerically on computer. For the numerical solution of 77 to (11), the unknown initial values have been computed by the method suggested by Jain and Menon 11 for linear boundary value problems. For this purpose, the Runge-Kutta-Gill integration scheme with a 
step size of 0.01 has been adopted . To ensure the accuracy of the numerical results so obtained, we have compared these results with the results obtained by exact solutions in some simple cases $(a=0)$ in which the numerical values of are readily available in Abramowitz and Stegun [10] and found that the numerical results obtained by both the methods are in good agreement.

\section{Skin FRICTION COEFFiCIENT}

The main physical quantities of interest are skin-friction coefficient

$$
C_{f}=\frac{\mu\left(\frac{\partial u^{\prime}}{\partial y^{\prime}}\right)_{y^{\prime}=0}}{\rho u_{0}^{\prime 2} / 2}
$$

which, in present case, can be expressed in the following form:

$$
C_{f}=\frac{1}{\sqrt{t}} \sum_{i=0}^{\infty}(m t)^{i} f_{i}^{\prime}(0) .
$$

Numerical values of functions $f_{i}^{\prime}, \theta(0)$ and $c(0)$ for $i=0$ to 2 obtained by numerical solution for $m=0.5 ; \operatorname{Pr}=0.72 ; a=-0.5,0,0.5 ; G r=5.0,10.0 ; G c=2.0,4.0$ and $S c=0.30,0.60,2.62$ are presented in Table 1 .

Table 1. Numerical values of wall shear stress functions $f_{i}^{\prime}, i=0,1,2$, wall temperature $\theta(0)$ function and concentration function $c(0)$ for $m=0.5$ and $\operatorname{Pr}=0.72$

\begin{tabular}{|c|l|l|l|l|l|l|l|l|}
\hline$a$ & $G r$ & $G c$ & $S c$ & $\Theta(0)$ & $c(0)$ & $f_{0}^{\prime}(0)$ & $f_{1}^{\prime}(0)$ & $f_{2}^{\prime}(0)$ \\
\hline \hline 0.5 & 5.0 & 2.0 & 0.30 & 0.68585 & 1.21744 & -1.83271 & 17.2102 & -3.78202 \\
\hline 0.5 & 5.0 & 4.0 & 0.30 & 0.68585 & 1.21744 & -1.83271 & 24.6409 & -5.34521 \\
\hline 0.5 & 10.0 & 2.0 & 0.30 & 0.68585 & 1.21744 & -1.83271 & 25.1569 & -5.08443 \\
\hline 0.5 & 5.0 & 2.0 & 0.60 & 0.68585 & 0.77613 & -1.83271 & 13.6166 & -2.88563 \\
\hline 0.5 & 5.0 & 2.0 & 2.62 & 0.68585 & 0.26603 & -1.83271 & 10.4687 & -2.28549 \\
\hline 0.0 & 5.0 & 2.0 & 0.30 & 1.04443 & 1.61496 & -1.12838 & 23.2893 & -4.88736 \\
\hline-0.5 & 5.0 & 2.0 & 0.30 & 1.81493 & 2.25838 & -0.57795 & 34.8856 & -6.98611 \\
\hline
\end{tabular}

Stokes 12 was the first to find an exact solution to the Navier-stokes equation for the case of flow past an impulsively started infinite horizontal plate in a viscous incompressible fluid. The Stokes problems which are of practical interest have been considered for single fluid. However, in nature, there is present a foreign mass like water vapor, carbon dioxide, helium etc. Hence we have to consider the flow of binary fluids. To study the effects of mass transfer on the flow, the value of the Schmidt number $S c$ is chosen to be $0.30,0.60$ and 2.62 which represent the diffusing chemical species of most common interest in air $(\mathrm{Pr}=0.72)$, namely $\mathrm{He}, \mathrm{H}_{2} \mathrm{O}$ and propyl benzene, respectively (Perry $[13]$ ). The value of the magnetic field parameter $m$ is chosen to be 0.5 in all the cases.

Figure 1 shows that the curve of skin-friction coefficient $C_{f}$ increases with time and become asymptotic as $t$ approaches 0.7 . It may be observed that $C_{f}$ increases with 


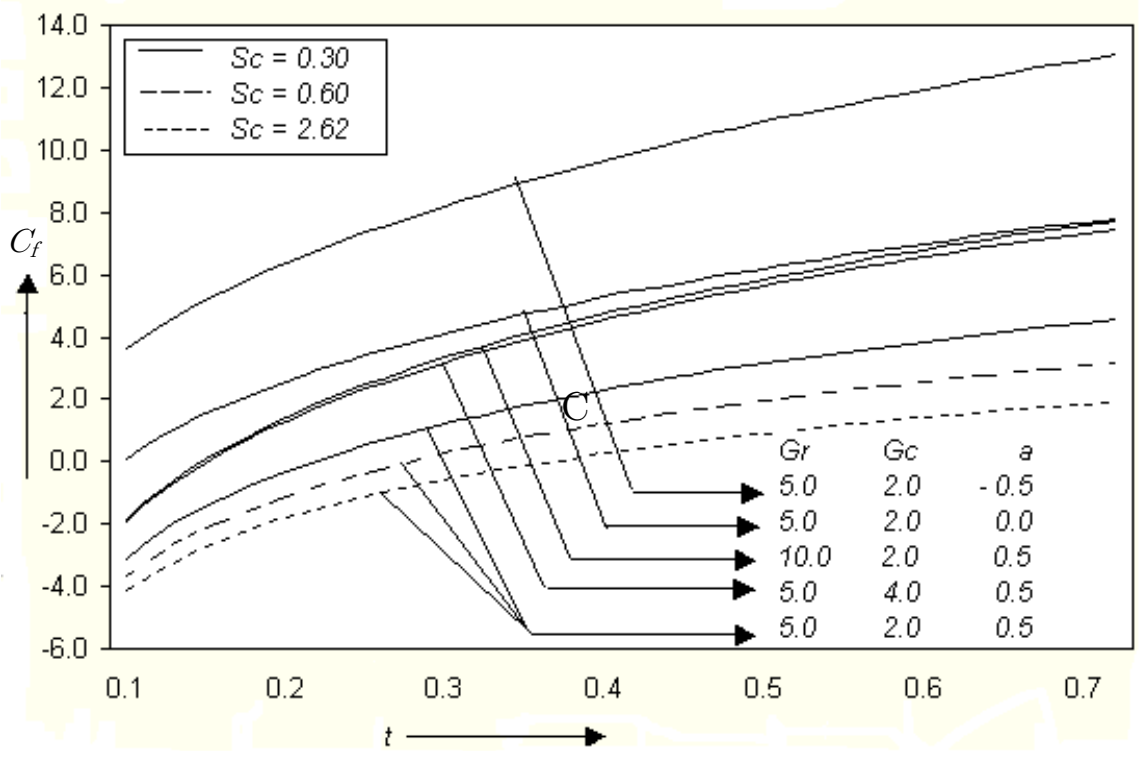

Figure 1. Variation of skin-friction coefficient with time at fixed values of $m=0.5$ and $\operatorname{Pr}=0.72$

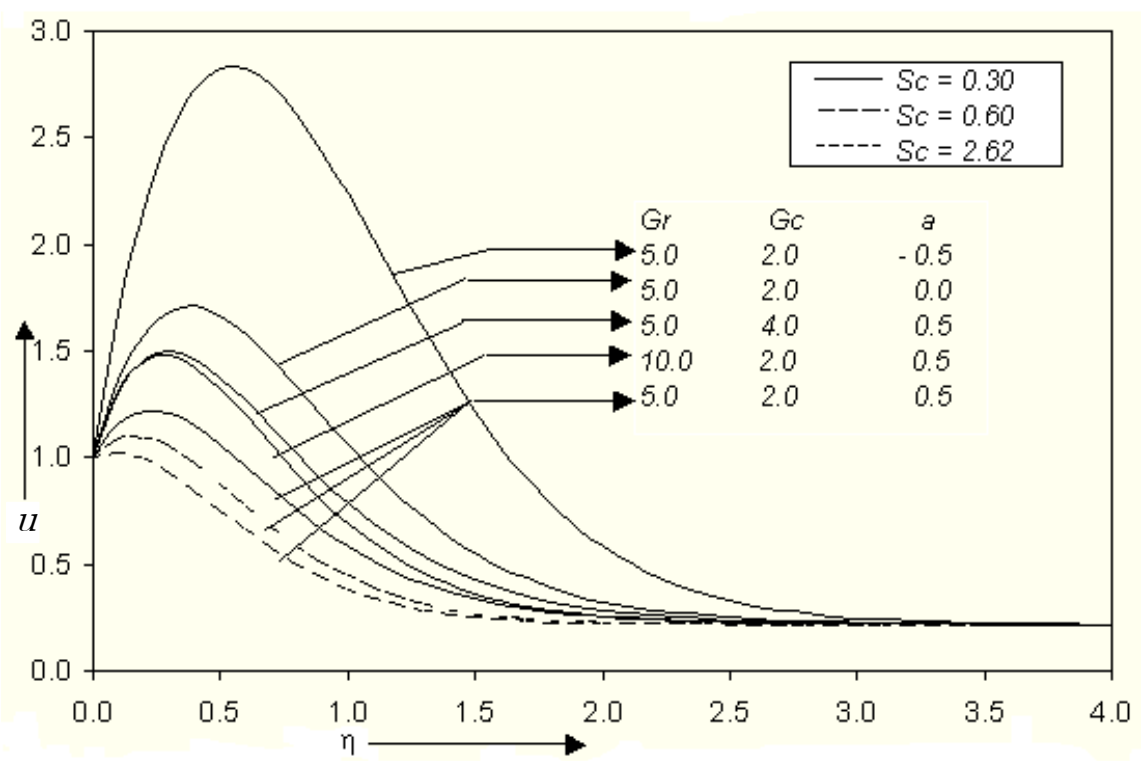

Figure 2. Velocity profile at fixed values of $t=0.5, m=0.5$ and $\operatorname{Pr}=0.72$ 


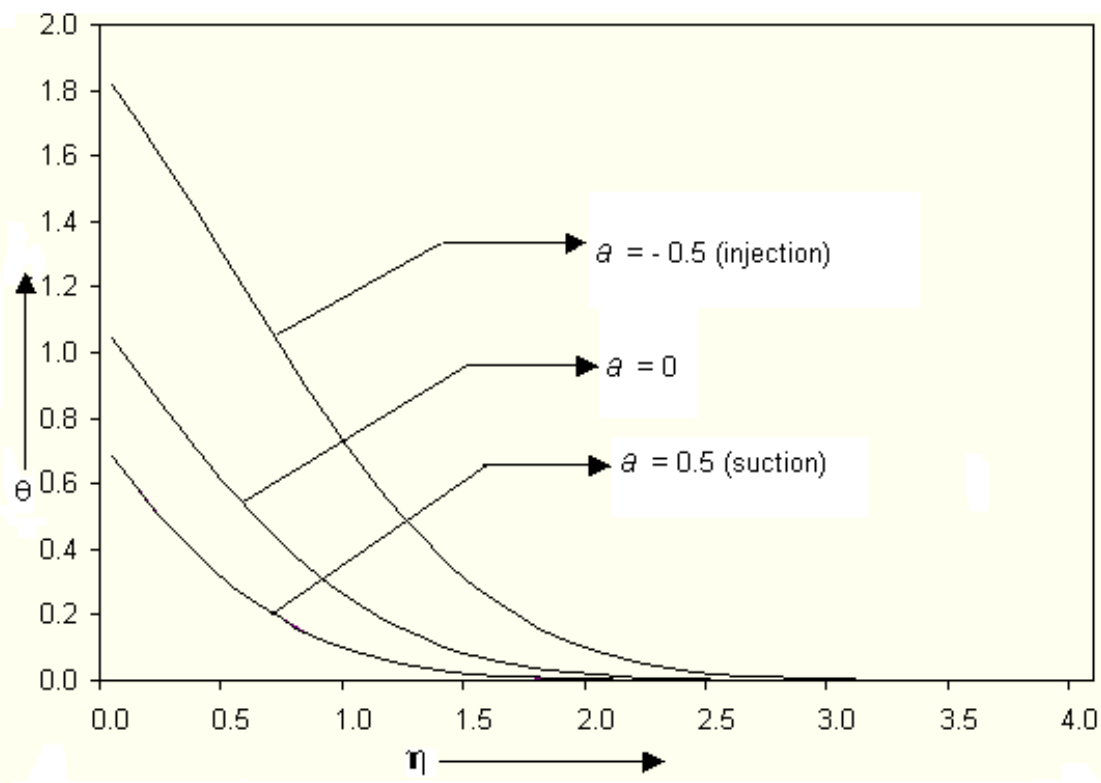

Figure 3. Temperature profile at fixed values of $t=0.5, m=0.5$ and $\operatorname{Pr}=0.72$

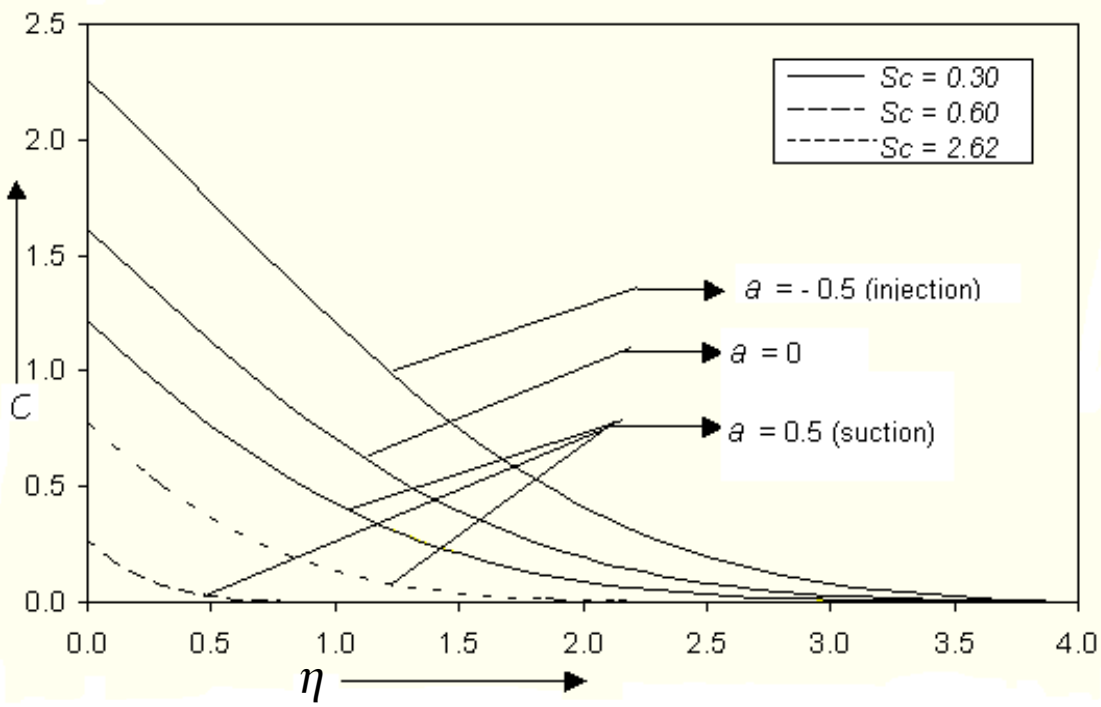

Figure 4. Concentration profile at fixed values of $t=0.5, m=0.5$ and $\operatorname{Pr}=0.72$ 
the Grashof number $G r$ and modified Grashof number $G c$ whereas it decreases as the Schmidt number $S c$ and the suction/injection parameter $a$ increases. Therefore the presence of foreign species reduces the skin friction.

In Figure 2, the velocity function $u$ is plotted against $\eta$. It is observed from the figure that the velocity decreases as $a$ or $S c$ increases whereas the velocity increases with $G r$ or $G c$ for a fixed $\eta$. Thus the presence of foreign species decreases the velocity boundary layer thickness. It may also be noted that for the large value of $\eta$, the velocity $u$ approaches 0.22 when $m=0.5$ and $t=0.5$ showing that the numerical solution satisfies the boundary condition 5 as $y \rightarrow \infty$ the exact value being 0.221199 . This also indicates the accuracy of the numerical solutions.

In Figure 3, the temperature profiles are plotted against the variable $\eta$. It is observed that as $a$ increases, the temperature function $\theta$ decreases for a fixed $\eta$.

In Figure 4 the concentration profiles are plotted against the variable $\eta$. It may be noted that the concentration decreases as the parameter $a$ or $S c$ increases for a fixed $\eta$.

\section{Conclusions}

1. Since the graph of $C_{f}$ becomes asymptotic for $t \geq 0.7$ in all the cases, it may be concluded that the flow is reduced to steadiness after time $t=0.7$.

2. Presence of foreign species reduces the skin friction and velocity.

3. The thermal boundary layer thickness decreases as the suction/injection parameter $a$ increases.

4. The concentration function decreases as the parameter $a$ increases for a fixed value of $S c$.

5. The velocity boundary layer thickness decreases as $a$, or increases whereas it increases with $G r$ or $G c$.

6. While the plate moves upward with the uniform velocity, the fluid near the plate moves with greater velocity due to convection, Particularly in the case of injection of the plate.

\section{REFERENCES}

1. Elbashbeshy, E. M. A: Heat and mass transfer along a vertical plate in the presence of magnetic field. Indian Journal of Pure and Applied Mathematics, 27(6), (1996), 621-???.

2. Pohlhausen, E: Der Wärmeaustausch zwischen festen Körpern und Flüssigkeiten mit kleiner Reibung und kleiner Wärmeleitung. Zeitschrift für Angewandte Mathematik und Mechanik, 1, (1921), 115-121.

3. Mohsin, M. A. and Mandal, A. C.: Mass transfer effects on the unsteady hydromagnetic free convection flow past an accelerated vertical porous plate. Journal of Physics D: Applied Physics, 18(7), (1985), L-53.

4. Dave, Abha, Bansal, J. L. and Jat, R. N.: Heat mass and momentum transfer in unsteady hydromagnetic free convection flow past an accelerated vertical plate. Proceedings of the National Academy of Sciences, India Section A: Physical Sciences, 50, (1990), 211-225. 
5. Pathak, G. AND TAK, S. S.: Heat and momentum transfer in free convection flow due to hot vertical accelerated plate in presence of transverse magnetic field. Journal of Ultra Scientist of Physical Sciences, 14(2), (2002), 240-247.

6. Soundalgekar, V. M.: Free convection effects on the Stokes problem for an infinite vertical plate. Journal of Heat Transfer (ASME), 99, (1977), 499-501.

7. TAK, S. S. and PATHAK, G.: Unsteady free convection along a hot vertical plate suddenly set in motion in presence of transverse magnetic field. Far East Journal of Applied Mathematics, 8(3), (2002), 169-182.

8. TAK, S. S. and Sisodia, G. S.: Viscous heating in unsteady free convection along a hot vertical plate suddenly set in motion in presence of transverse magnetic field. Proc. Int. Conf. SSFA, VOL . II, (2002), 65-73.

9. Gebhart, B.: Heat Transfer. Second Edition, McGraw-Hill, 1971.

10. Abramowitz, M. and Stegun, I. A.: Hand Book of Mathematical Functions with Formulas, Graphs and Mathematical Tables, Dover, Tenth Edition, 1972, p. 299.

11. Jain, A. C. and Menon, K. R.: Görtler's series solution of laminar boundary layer equations with suction or injection. Zeitschrift für Angewandte Mathematik und Mechanik. 51, (1971), 245-254.

12. Stokes, G. G.: On the effect of the internal friction of fluids on the motion of pendulums. Proceedings of the Cambridge Philosophical Society, 9, (1851), 8-106.

13. Perry, J. H.: Chemical Engineers Handbook. 4th Edition, McGraw-Hill, 1963. 